\section{Liver from bone}

First, blood cells from brain cells and myocytes from bone marrow. Now researchers at New York (NY) and Yale (New Haven, CT) universities have shown that liver cells can also arise from bone marrow, challenging the convention that adult cells are irreversibly stamped with their destiny. Using female mice in which bone marrow had been completely destroyed by irradiation, the scientists introduce bone marrow cells from age-matched male mice and, over the following six months, tracked the appearance of mature liver cells in the recipients that bore the XY chromosomal signature of male cells. They discovered that up to $2.2 \%$ of mature liver cells were derived from the donated marrow. It sets up the idea that there's a steady physiological process," says Neil Theise, who headed the research. "If we can isolate these cells, we have targets for gene therapy and the possibility of stem cell transplantation, rather than whole-organ transplantation," he believes. The findings are reported in Hepatology (31, 235-240, 2000).

\section{Sugary synthetic genes}

By constructing synthetic genes to express predicted glycosylation consensus sequences, researchers at Ohio University (Athens, $\mathrm{OH}$ ) and Michigan State University (East Lansing, MI) have identified a simple glycosylation code in plants. Despite the central importance of glycosylation in the biogenesis of many plant proteins, the mechanisms controlling this process have remained poorly understood. In the new work, the researchers designed two synthetic genes encoding putative glycoproteins. One protein carried predicted polysaccharide addition sites, while the other, based on the consensus sequence of gum arabic, contained arabinogalactan and arabinooligosaccharide addition sites. The synthetic genes were expressed in tobacco cells, and the resulting proteins were glycosylated as predicted, indicating that the cellular glycosylation machinery follows a simple code in adding these sugars to protein modules. "We have demonstrated that a synthetic gene approach is effective for testing glycosylation codes and the specificities of glycosyl transferases," says Marcia Kieliszewski, senior author on the study. The team also hopes to use the conserved glycosylation code to manufacture novel glycoproteins in crop plants like tobacco and tomato.

Research News Briefs written by Alan Dove and Keely Savoie.

\title{
Stem cell nuclear transfer
}

The successful generation of live mice from cloned mouse embryonic stem (ES) cells opens up new possibilities for the creation of transgenic mice (Proc. Nat. Acad. Sci. USA 96, 14984-14989, 1999). Previously, viable clones had only been obtained from freshly isolated, ill-defined donor cells. Now, researchers at the University of Hawaii and Rockefeller University have obtained live-born mice by microinjecting ES cells into enucleated metaphase-II oocytes,

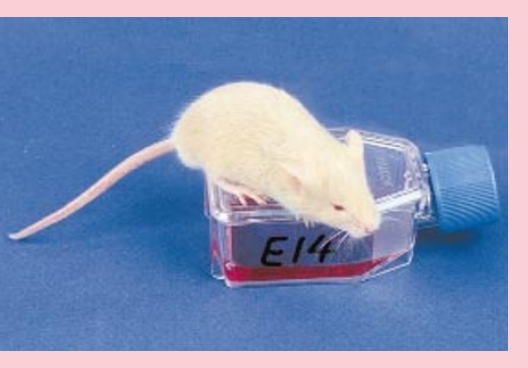
activating division using strontium ions

(sometimes with cytochalasin B to prevent aberrant cytokinesis), and implanting the cells into pseudopregnant mice. The ES cells used as nuclear donors were either in G1 or post$S$ phase. According to Rockefeller University's Peter Mombaerts, a coauthor on the study, the success of the approach shows that "the dogma of the need for quiescence is not universally true." From a practical standpoint, the ability to clone genetically manipulated ES cells could transform transgenic research: "If we could go directly from a cell in culture in a dish to a live animal, it would skip two generations," he says. The ability to use established cell lines should also allow future research to be standardized and replicated from laboratory to laboratory. Mombaerts says the next step in standardization is to publish a detailed protocol for cloning.

In a major technical accomplishment, a team of researchers has inserted three genes into rice to allow the plant to produce beta carotene, the precursor required for animals to make vitamin $\mathrm{A}$. The work, reported in Science $(287$, $303-305,2000$ ) is a significant step beyond the single-gene insertions typical of agricultural biotechnology so far. The three enzymes added to the rice constitute a complete biosynthetic pathway for beta carotene, and the resulting golden-colored grains should provide a good source of vitamin A for some of the estimated 124 million children worldwide whose diets are deficient in this essential nutrient. "There are other crops for which the same technology will be applied and experiments and collaborations are already starting. These include wheat, African white maize, barley, banana, cassava, sweet potato," says Ingo Potrykus, a researcher at the Institute for Plant Sciences at the Swiss Federal Institute of Technology (Zurich, Switzerland) and senior author on the paper. Potrykus adds that the genetically enriched rice might also be useful in preventing macular degeneration and maintaining eyesight in the elderly in developed countries. The scientists plan to

distribute the transgenic seeds free of charge for non-commercial use in developing counveloping countries and in rice breeding programs.

\section{Monkey around with clones}

A major bottleneck in translating preclinical findings into clinical results is the difficulty of performing experiments in nonhuman primates. While genetically similar inbred mice can be produced in large numbers, "monkeys almost never have twins, so the opportunity to study genetically identical offspring is not available naturally," says Gerald Schatten, a researcher at Oregon Health Sciences University. To address this problem, Schatten and his colleagues have developed a technique for splitting the cells of an early monkey embryo to produce multiple identical animals. The new method, described in Science (287, 317-319, 2000), involves splitting the cells of a rhesus monkey embryo at the 8-cell stage and separating them into four 2-cell embryos that are then implanted in surrogate mothers. In the initial experiment, the researchers achieved a $31 \%$ success rate in establishing pregnancies, though only one of the four pregnancies resulted in a live birth. The baby monkey, named Tetra, appears to be developing normally. By splitting and implanting larger numbers of embryos, the team expects to be able to produce identical twins, triplets, and quintuplets. According to Schatten, "this would permit the investigation of the environmental causes of diseases versus the genetic basis." It may also provide a useful model system to bridge the gap between mice and humans. 